\title{
LEUKOCYTE CONTRIBUTIONS TO THE INDUCTION AND RESOLUTION OF ACUTE INFLAMMATION IN TELEOST FISH
}

\author{
$\underline{\text { Daniel R. Barreda }}^{1,2, \$}$, Jeffrey J. Havixbeck ${ }^{1}$, Michael J. Trites ${ }^{1}$, Michael E. Wong1 \\ ${ }^{1}$ Department of Biological Sciences and ${ }^{2}$ Department of Agricultural, Food and Nutritional \\ Science, University of Alberta, Edmonton, AB, Canada, T6G2P5
}

Acute inflammation drives early engagement of invading pathogens and contributes to the development of adaptive responses. This presentation will summarize recent experiments from our lab looking to define the contributions of leukocytes to the induction and resolution of acute inflammation in teleost fish. Using combinatorial cellular and molecular approaches we find that classic effectors such as macrophages and neutrophils elicit potent antimicrobial responses early in the acute inflammatory process.

Interestingly, both leukocyte subsets also contribute to the resolution of inflammation. A timely shift in their phenotype, from pro-inflammatory to pro-resolving is largely driven by interactions with apoptotic cells at the site of inflammation. Examination of blood and hematopoietic compartments shows a tight correlation between hematopoietic blood cell production, their migration through circulation and discrete functional events at the infection site. Molecular effectors like transferrin also appear to play an important role in vivo, as previously suggested by in vitro experiments from other research groups. These molecules are also helping to better define the various phases of this acute inflammatory response. In all, we find that the capacity of leukocytes to polarize towards pro-inflammatory or resolution roles existed before the divergence of fish and tetrapods, over 450 million years ago. We also find unique features within the teleost fish group, such as their capacity to respond efficiently to pathogen challenge despite maintaining much lower numbers of circulating neutrophils $(<5 \%)$.

KEYWORDS: acute inflammation, bony fish, leukocyte, immune response, evolution

${ }^{\S}$ Corresponding author:

Dr. Daniel Barreda

Tel.: 1(780)492-0375

E-mail address: d.barreda@ualberta.ca 